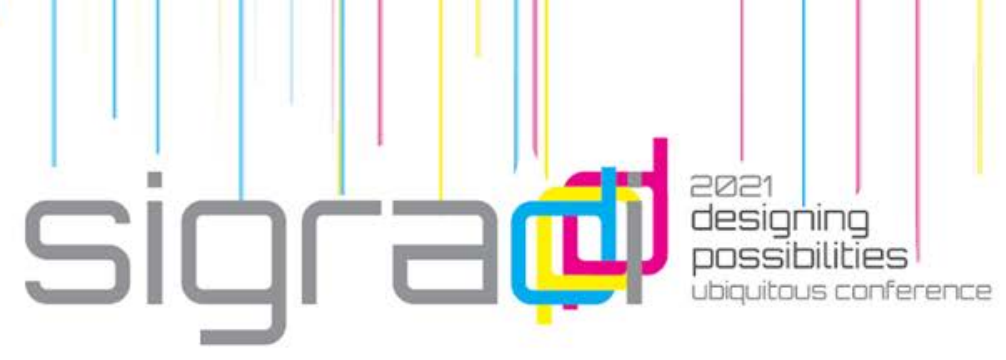

\title{
Energy-Based Design: A Digital Design System for the Design of Energy-Harvesting Building Envelopes
}

\author{
Daniel Mateus ${ }^{1}$, José Pinto Duarte², Luís Romão ${ }^{1}$ \\ ${ }^{1}$ CIAUD, Lisbon School of Architecture, University of Lisbon, Portugal \\ mateus.dani@gmail.com \\ Iromao@fa.ulisboa.pt \\ ${ }^{2}$ SCDC, College of Arts and Architecture, The Pennsylvania State University, USA \\ ixp400@psu.edu
}

\begin{abstract}
The goal of the research described in this paper is to address climate change by promoting the production of renewable energy in building envelopes, which are exposed to solar radiation. It proposes an energy-based design paradigm, where energy processes shape the building form, and a digital design system for building envelopes that considers the trajectory of sunrays. The goal is to create envelopes that are efficient in harvesting solar energy, enabling them to produce the electricity that buildings consume. To operationalize the proposed digital design system, a building envelope grammar is developed and implemented in a software called LIDIA to be used by architects in design process to generate solutions with improved energy production performance. The efficiency of the resulting solutions, the effectiveness of LIDIA and, therefore, the validity of the proposed paradigm, is demonstrated with the design of envelope solutions for single family houses.
\end{abstract}

Keywords: Energy-based design, Building envelope, Building envelope grammar, Shape grammar, LIDIA software.

\section{Introduction}

The energy-based design paradigm resides in the idea that the shape of things in nature and in the universe comes from formation processes in which energy plays a fundamental role. Carl Sagan wrote that the passage from the Chaos of the Big Bang to the Cosmos that we are beginning to know is the most awesome transformation of matter and energy that we have been privileged to glimpse. (Sagan, 1980) Energy-based design applied to buildings intends to mirror the processes of formation of things existing in nature and in the 
universe. To illustrate and test the proposed energy-based paradigm, this paper describes a digital design system for building envelopes. Buildings sector account for $36 \%$ of the total value of energy consumption worldwide (United Nations Environment and International Energy Agency, 2017). In this context, it worthwhile to promote the production of renewable energy in building envelopes exposed to solar radiation. The goal is for buildings to achieve energy self-sufficiency, making them producers, in addition to consumers, of energy.

The proposed digital design system adapts the shape of envelopes to the trajectory of sunrays, thus allowing to increase the capacity of envelopes to harvest solar energy, with the aim of transforming it into electricity using photovoltaic technology. Shape grammars (Stiny, 1980) are the formalism used to encode the design system. A key reference in the use of shape grammars in energy performance-related research is the work of Granadeiro (2013), which aimed to establish methods to support decisions at early stages of design, related to the design of the building envelope, for residential buildings. Granadeiro's work uses a shape grammar to generate different functional and compositional solutions for Frank Lloyd Wright's prairie houses, and genetic algorithms to find solutions in the universe of solutions defined by the grammar that have lower energy consumption values. The design system described in this paper builds on the research developed by Granadeiro, relating shape grammars with energy and with the energy performance of buildings. However, it uses shape grammars to generate shapes for the buildings envelope that are more efficient in harvesting solar energy to produce electricity. Therefore, it addresses energy production more than energy consumption, although values of energy consumption are also provided as feedback.

\section{A digital design system for building envelopes}

\subsection{The Building Envelope Grammar}

The proposed digital design system is based on a grammar for building envelopes, which generates energy-harvesting building envelopes for single family houses with different programs in different site contexts. It is a generic discursive grammar that uses the concepts of shape grammar (Stiny, 1980), description grammar (Stiny, 1981), discursive grammar (Duarte, 2001) and generic grammar (Beirão, 2012; Benrós, 2018). The building envelope grammar consists of six shape grammars and one description grammar, as follows: the shape grammar $A$ of the building envelope, the shape grammar $L$ of the urban lot, the shape grammar $E$ of the isolated envelope, the shape grammar S of the envelope surfaces, the shape grammar $\mathrm{F}$ of the envelope photovoltaic elements, and the shape grammar $\mathrm{O}$ of the operators, which allows the use of a diverse range of initial shapes and the generation of a diverse set 


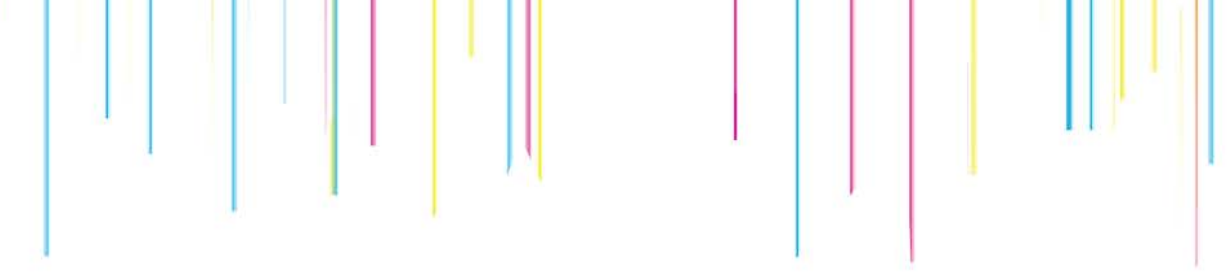

BUILDING ENVELOPE GRAMMAR (Shape Grammar A):

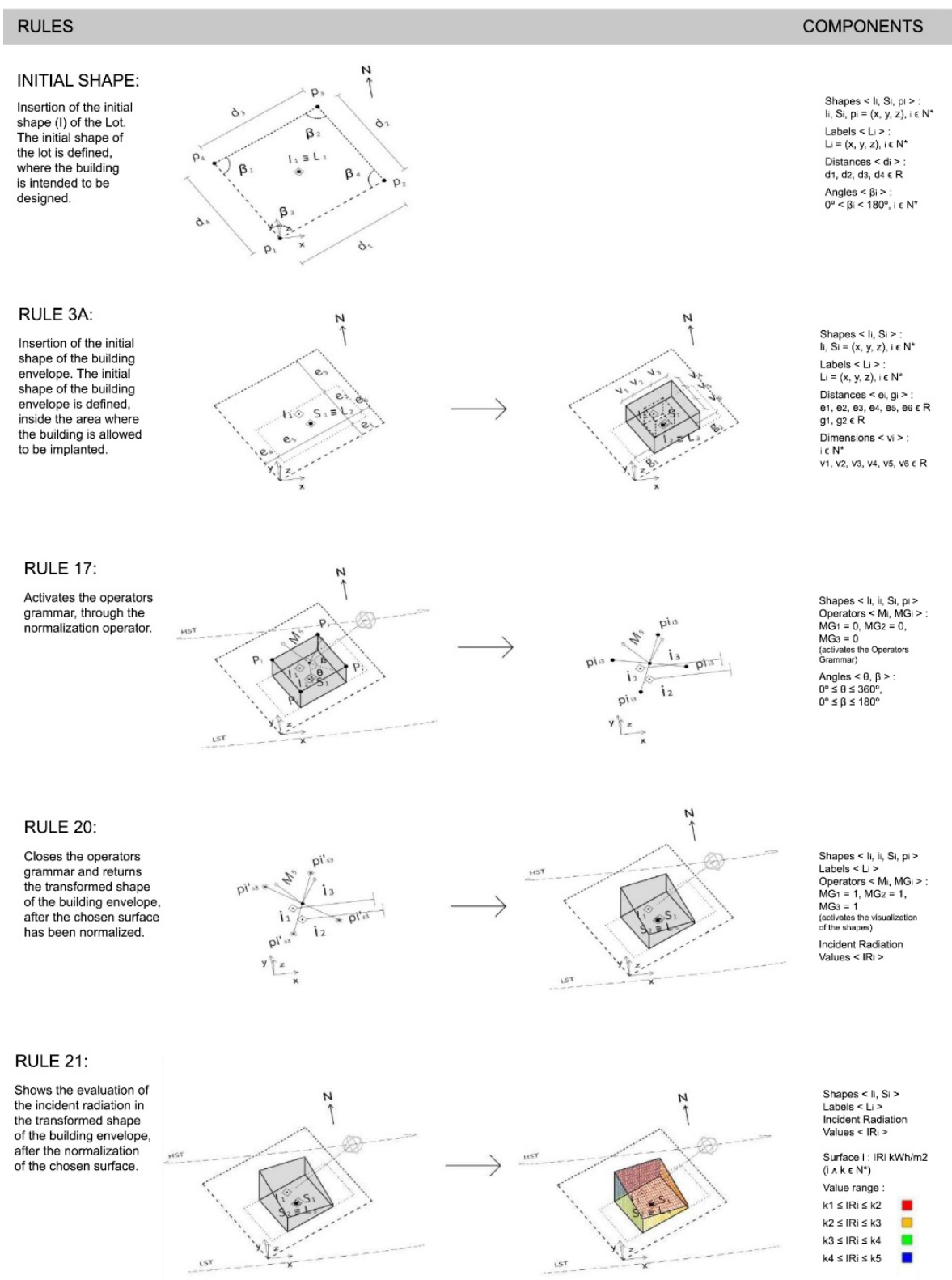

Figure 1. Example of rules from the compound generic Building Envelope Grammar. The rules in question are presented in a simplified format, showing only the part of the rules related to shape grammar $A$, which brings together the remaining shape grammars. The building envelope grammar includes five other shape grammars and one description grammar (not shown). Source: The authors, 2021. 


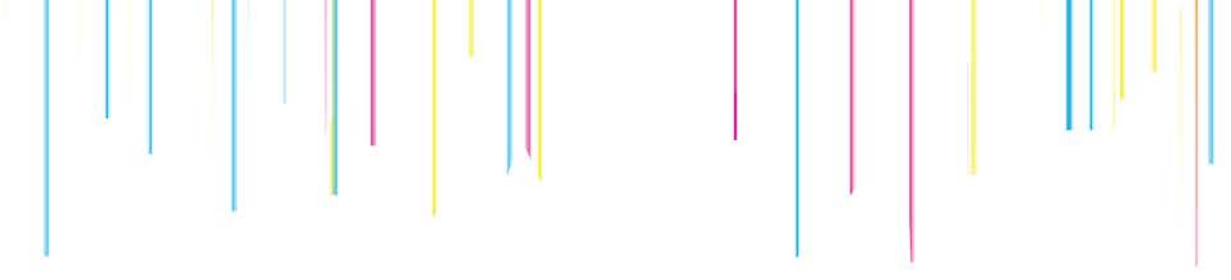

RULE 20:

A - Shape Grammar of

the Building Envelope

Grammar type

Shape

Algebra <Shapes, Labels>

$<\mathrm{U}_{33} \mathrm{~V}_{03}>$

Type of view :
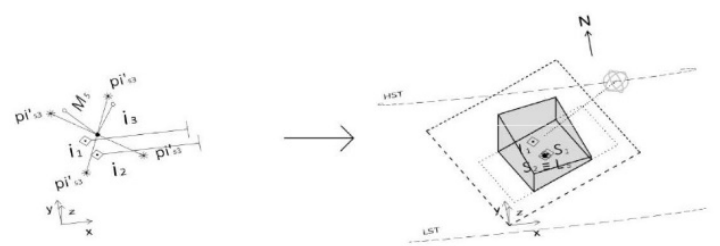

Shapes $\langle\mathrm{l}, \mathrm{i}, \mathrm{S}, \mathrm{p} \mid\rangle$

Labels $\left\langle L_{i}>\right.$

Operators $<\mathrm{Mi}_{i}, \mathrm{MG}_{i}$
$\mathrm{MG}_{1}=1, \mathrm{MG}_{2}=1$

$\mathrm{MG}_{3}=1$

(activates the visualization

of the shapes)
Incident Radiatio

Incident Radiation
Values $\left\langle\mid R_{i}\right\rangle$

\section{L - Shape Grammar \\ of the Lot \\ Grammar type \\ Shape \\ Algebra <Shapes, Labels> \\ $<\mathrm{U}_{23} \mathrm{~V}_{03}>$ \\ Type of view :}
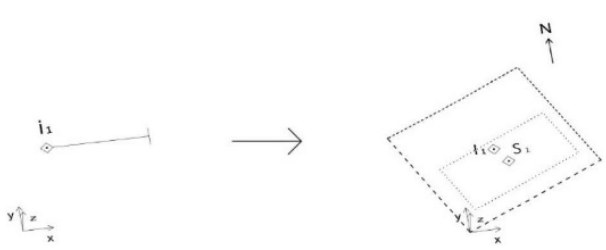

Shapes $\left\langle\mathrm{H}_{\mathrm{i}} \mathrm{i}, \mathrm{S}_{\mathrm{i}}\right\rangle$

$\mathrm{MG}_{1}=1$

(activates the visualization

of the shapes)

E - Shape Grammar of the Isolated Envelope Grammar type :

Shape

Algebra <Shapes, Labels :

$<\mathrm{U}_{33} \mathrm{~V}_{03}>$

Type of view
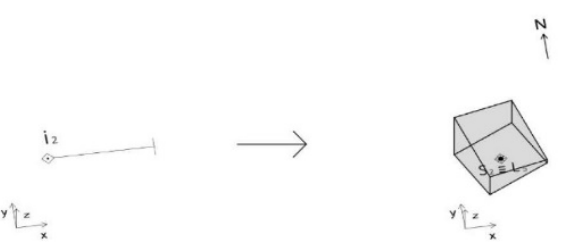

Shapes $\left\langle\mathrm{i}_{i} \mathrm{i}, \mathrm{S}_{\mathrm{i}}\right\rangle$

$\mathrm{MG}_{2}=1$

activates the visualization

of the shapes)

Values $\left\langle\mathbb{R}_{i}\right\rangle$

S - Shape Grammar of the Surfaces

Grammar type :

Shape

Algebra <Shapes, Labels $>$

$<\mathrm{U}_{23} \mathrm{~V}_{03}>$

Type of view:
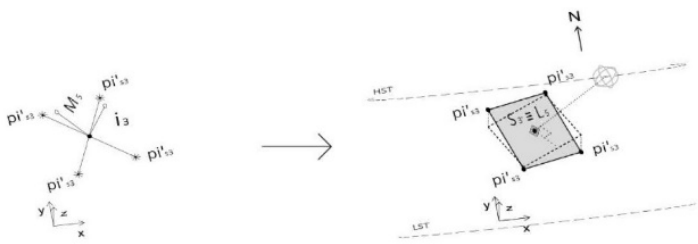

Shapes $\left\langle\mathrm{li}_{\mathrm{i}} \mathrm{i}, \mathrm{Si}, \mathrm{pi}\right\rangle$

Operators $\left\langle M_{i}, M G_{i}>\right.$ :

$\mathrm{MG}_{3}=1$

(activates the visualization

of the shapes)

Incident Radiation

O - Shape Gramma

of the Operators

Grammar type :

Algebra <Shapes, Labels>

$<\mathrm{U}_{23} \mathrm{~V}_{03}>$

Type of view:

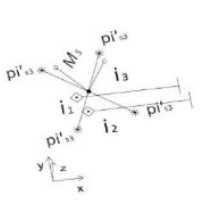

Shapes $<$ ii $>$

Operators $<M, M G i$ >

$\mathrm{MG}_{3}=1$

(activates the visualization

of the shapes)

Incident Radiation
Values $\left\langle\mathbb{R}_{i}\right\rangle$

\section{D - Descriptive Grammar}

Grammar type

\# $\mathrm{cl} 1.1$ \# cl1.2 \# cl2.1 \# cl2.2 \# cl3.1 \# cl3.2 \# cl4.1 \# cl4.2 \# c15.1 \# c15.2 \# cl6.1 \#

cl6.2 \# cl7.1 \# cl7.2 \# cl8 \# c19.1 \# cl9.2 \# cl10.1 \# cl10.2 \# cl11 \# ca1.1 \# ca1.2 \#

$\mathrm{ca} 2$ \# ca3 \# ca4 \# ca5 \# p1 \# p2 \# p3.1 \# p3.2 \# eic1 \# eic2 \# eic3 \# eic4 \# eic5 \#

eic6.1 \# eic6.2 \# eic6.3 \# eic6.4 \# eip1 \# eip2.1 \# eip2.2 \# eip2.3 \# eip2.4 \# eip2.5

\# еip3.1 \# еip3.2 \# еip3.3 \# eip3.4 \# eip3.5 \#

Figure 2. Rule 20 of the Building Envelope Grammar, in full format. Rule 20 represents one step in the normalization operation, which makes an envelope surface perpendicular to a chosen position of the solar trajectory throughout the year. Source: The authors, 2021. 


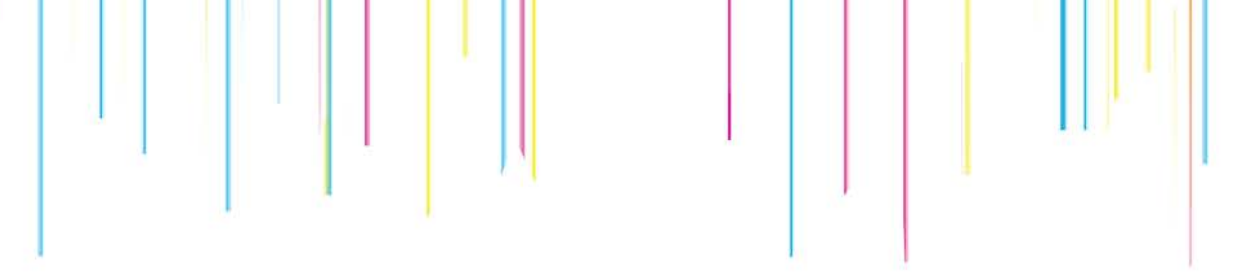

of shapes for the building envelope. It also includes the descriptive grammar D of the context, program, and interior space of the building.

The building envelope grammar includes shape transformation rules that perform operations on the building envelope, namely, rotation, normalization, division, and evaluation of the incident solar radiation. Normalization makes a surface of the envelope perpendicular to the sunrays, considering a chosen sun position from the solar trajectory throughout the year. The process of evaluation of the incident solar radiation and consequently of energy production in the building envelope is thus integrated into the building envelope grammar. After applying each shape transformation rule, the amount of harvest energy is recalculated using a solar radiation evaluation software. The rules were designed to increase the performance of the envelopes in harvesting solar radiation as they are applied, but they do not guarantee an optimized result.

The building envelope grammar is also a compound grammar, whose rules use all their sub-grammars, except the shape grammar of the photovoltaic elements, which has not been developed yet. Figure 1 shows an initial shape and a sample of the grammar rules in a simplified format, showing only the shape grammar $\mathrm{A}$ of the building envelope. Figure 2 shows the transformation Rule 20 in full format, also as an example. The remaining rules of the grammar, as well as the complete description of its features, will be presented in a future paper.

\subsection{The LIDIA software}

LIDIA stands for Language Interface for the Development of Intelligent Algorithms and is a software prototype created specifically for the building envelope design system. It implements the generic envelope grammar and allows the development of specific grammars that can be used to generate efficient envelope solutions in capturing solar radiation.

LIDIA is composed of visual programming components designed specifically for Grasshopper either by combining existing Grasshopper components or by programming new ones in the Grasshopper Python Script Editor. In addition to support the development of different specific grammars, LIDIA also supports the exploration of different design solutions within each specific grammar through a simplified graphical user interface. The interface also allows users to visualize the incident solar radiation and the estimated electrical energy production. Figure 3 illustrates the use of the LIDIA in a design session, including the main interface, where different solutions for the envelope can be explored, and the secondary interfaces, where it is possible to visualize and compare the performance of different design solutions. It is also possible to view one of the solutions in Rhinoceros 3D, which is active through Grasshopper. 


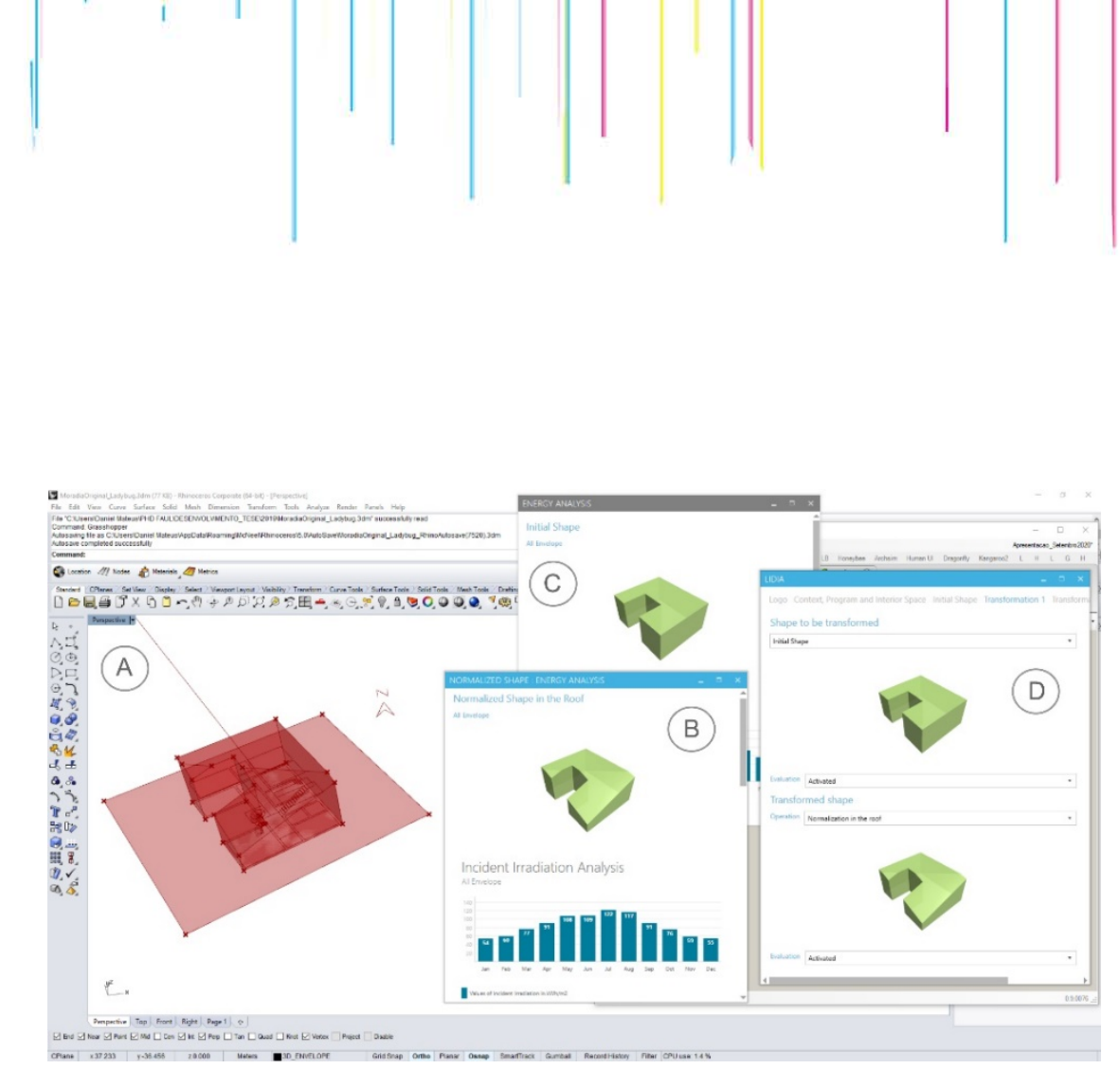

Figure 3. Use of LIDIA's interface in the design of a building envelope: $(A)$ image of the building in Rhinoceros 3D; (B) window with the energy production of a normalized surface on the roof of the building; $(C)$ window with the energy production of the initial shape of the building envelope; (D) main window of LIDIA, used to transform the shapes of the building envelope and activate the secondary windows with the energy production values of the resulting envelope designs. Source: The authors, 2021.

\section{Application of the Building Envelope Grammar and of LIDIA in the envelope design of two single family houses}

\subsection{Designed solutions for the envelope}

To demonstrate the feasibility of the building envelope grammar and LIDIA, a case study was developed, by applying them to the envelope design of two single family houses with two (T2) and five (T5) bedrooms. A vacant urban lot, located in the parish of Belém, in the city of Lisbon, Portugal, was chosen for the development of the case study. Two distinct design strategies were considered, elaborating two types of shapes: (1) the static envelope shape; and (2) the dynamic envelope shape. The difference between the two is that in the static envelope, the shape of the house's envelope is fixed. In the dynamic envelope, the shape of the envelope varies throughout the year, so that its surfaces are more efficiently exposed to sunrays. Figures 4 and 5 illustrate design solutions generated using LIDIA. In this paper, due to space limitations, only the static shapes A, D, E and G and the dynamic shape (Shapes I1, I2, I3 and 14) of house T5 are presented. The remaining static and dynamic shapes will be shown in a future paper. Incident radiation values per $\mathrm{m}^{2}$ and the total incident radiation values are shown both for the whole envelope and for the roof surfaces of the envelope. 

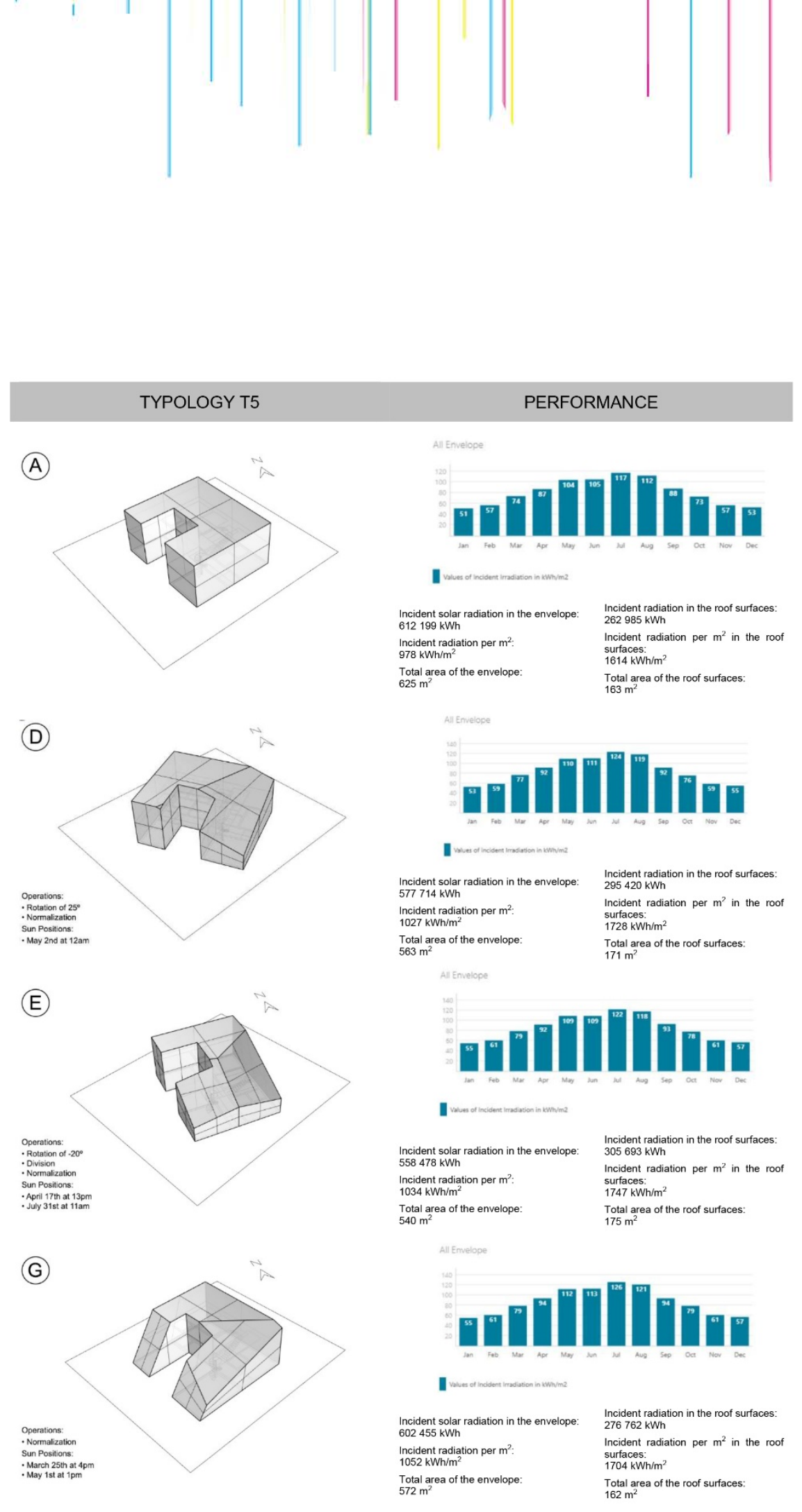

Figure 4. Static shapes of the five-bedroom typology (T5) building envelope: (A) initial shape, (D) rotated and normalized shape; (E) rotated, divided, and twice normalized shape on the roof; and (G) normalized shape on the roof and on the facade. Source: The authors, 2021. 

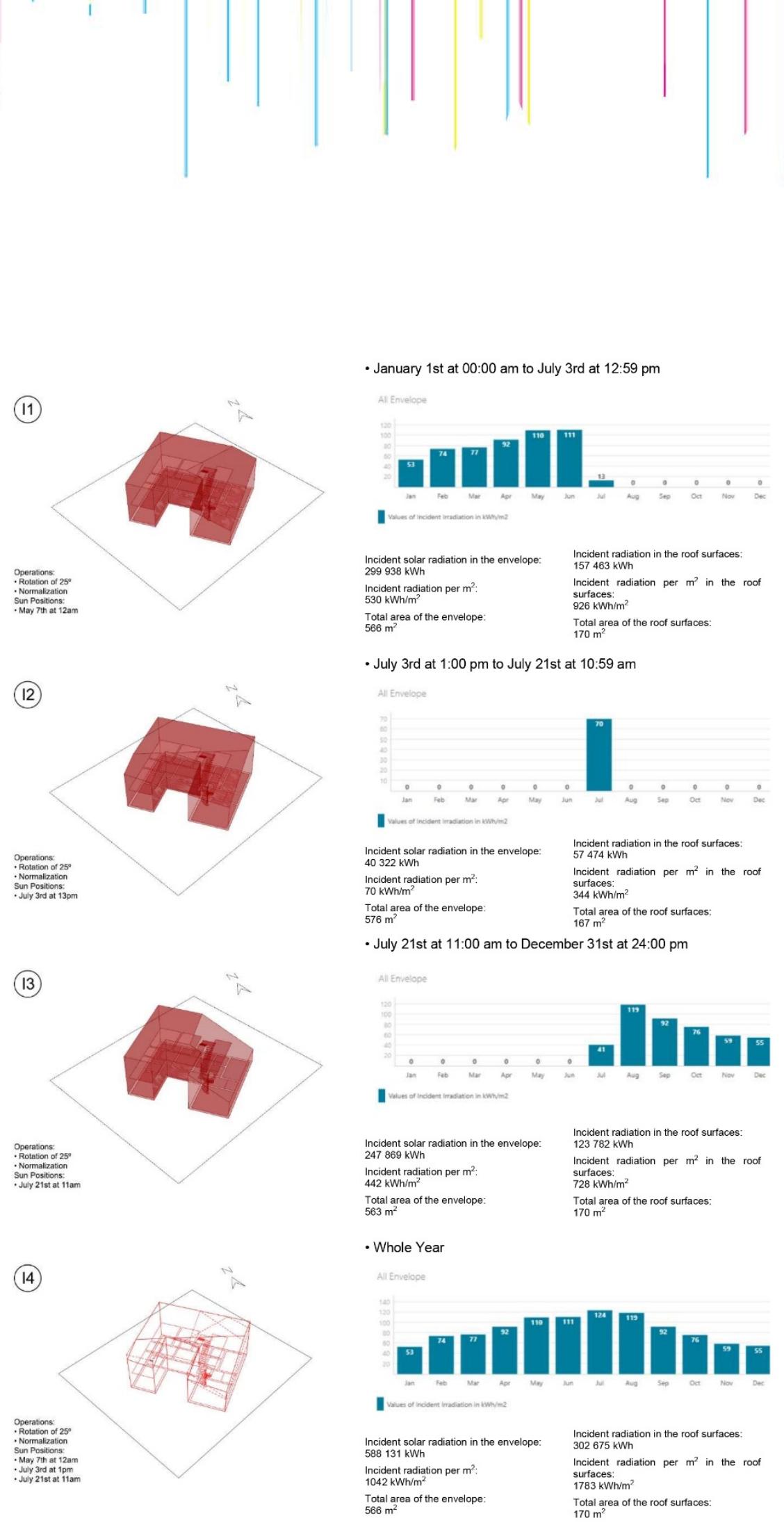

Figure 5. Dynamic shapes of the five-bedroom typology (T5) building envelope: (I1) normalized shape for May 7th at 12:00 am; (I2) normalized shape for July 3rd at 1:00 pm; (I3) normalized shape for July 21st at 11:00 am; and (14) the whole shape, composed by the shapes I1, 12 and I3. Source: The authors, 2021. 


\subsection{Analysis of results}

The results obtained after different shape transformation operations, show that:

- The shape transformation operations of the building envelope grammar improve the energy efficiency of the building envelopes by increasing the amount of incident solar radiation per $\mathrm{m}^{2}$ on the envelope. Different sequences of the rotation, normalization and division operations lead to different shapes and different energy performance values of the building envelope.

- The dynamic shape performs well in terms of incident solar radiation per $\mathrm{m}^{2}$ and total incident radiation, both for the entire envelope and for the roof surfaces, compared to static shapes, including the rotated and normalized shape that serves as a basis for the elaboration of the dynamic shape.

Table 1. Energy performance of the five-bedroom (T5) typology house.

\begin{tabular}{llcc}
$\begin{array}{l}\text { Typology T5 } \\
\text { (roofs) }\end{array}$ & $\begin{array}{l}\text { Value of electricity } \\
\text { produced in the } \\
\text { roof }\end{array}$ & $\begin{array}{l}\text { Value of electricity } \\
\text { consumed in the } \\
\text { building }\end{array}$ & $\begin{array}{c}\text { Energy production } \\
\text { performance } \\
\text { compared to the } \\
\text { initial shape }\end{array}$ \\
\hline $\begin{array}{l}\text { Initial shape } \\
\text { (Shape A) }\end{array}$ & $58383 \mathrm{kWh} /$ year & $8537 \mathrm{kWh} /$ year & ----------- \\
\hline $\begin{array}{l}\text { Rotated and } \\
\text { normalized shape } \\
\text { on the roof } \\
\text { (Shape D) }\end{array}$ & $65583 \mathrm{kWh} /$ year & $8537 \mathrm{kWh} /$ year & +7 200 kWh/year \\
$+12,3 \%$
\end{tabular}

. Source: The authors, 2021.

Table 1 presents an estimate of the produced electricity values, obtained from the total value of solar radiation incident on the roof surfaces, considering an efficiency rate of $22,2 \%$, which is the efficiency rate of the photovoltaic panels with the best performance existing in the Portuguese market in 2018 (Reis, 2018). Table 1 also includes an estimative of the amount of electricity consumed in the building, which is calculated based on the value of household electricity consumption per person indicated in the PORDATA database 


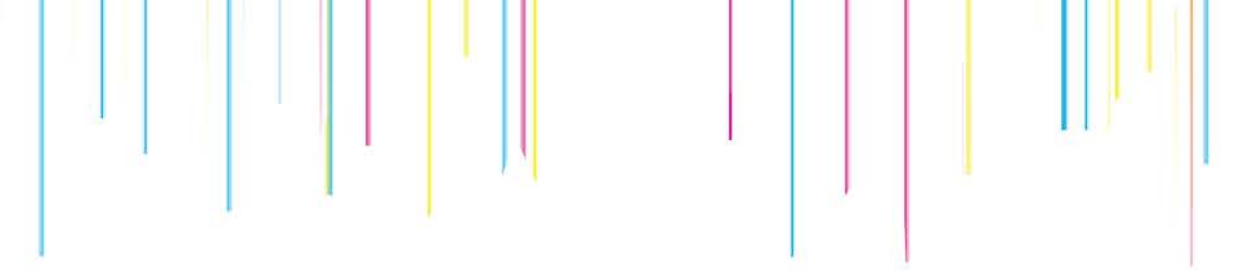

(PORDATA, 2019). Finally, Table 1 also shows the performance in terms of energy production of the designed solutions compared to the initial shape.

\section{$4 \quad$ Discussion and Conclusions}

\subsection{Discussion}

The results obtained in the envelope design show that the proposed building envelopes design system can be used successfully in the design of efficient energy-harvesting building envelopes. However, it is important to discuss how the digital design of building envelopes is related to other features of the architectural design of buildings, namely:

(1) Building materials and their influence on the shape of the building envelope: Two methodologies can be adopted for the inclusion of materials in the building envelope design process. The first methodology consists of choosing and applying suitable materials for the construction of the designed shapes, considering their efficiency in capturing sunrays. The second methodology consists of establishing an initial shape that is defined according to the characteristics of the materials, which are chosen a priori in the initial phase of the design. The initial shape is then transformed by applying the rules of the building envelope grammar to become efficient in capturing the sunrays. Our goal is to integrate these two methodologies in future developments of the building envelope grammar and LIDIA.

(2) Quality of the interior space of buildings: Currently, the building envelope grammar and LIDIA allow the user to explore the volumetric configuration of the interior space of buildings through the transformation of the envelope shape, while the building layout remains unchanged. Our goal is to integrate both so that the layout can be changed during the design of the building envelope, thus supporting the simultaneous exploration of interior space design.

(3) Envelope aesthetics: Our goal is also to develop the grammar and LIDIA so they can allow users to explore more broadly the aesthetics of the envelope's roof and facades by applying different geometric patterns and textures, which may or may not be associated with efficiency in capturing sunrays. 


\subsection{Conclusion}

This paper dwells on a new energy-based paradigm for the design process. More specifically it describes a system to define the shape of the building envelopes according to the trajectory of sunrays, thus increasing the capacity of the envelopes to harvest solar energy. The goal is for buildings to achieve energy self-sufficiency by making them energy producers, not just consumers. The system is based on a generic grammar called building envelope grammar, that is implemented in Rhino and Grasshopper, in a software prototype called LIDIA, which can be used to create specific grammars. These can then be used to create design solutions with increased energy harvesting performance. The effectiveness of LIDIA is discussed and demonstrated with its use in the design of envelopes for single-family houses, thereby demonstrating the potential of the proposed energy-based design paradigm.

LIDIA can be used by designers in the design of building envelopes with increased performance. The designer chooses which and where rules are applied, and the system provides feedback regarding the performance of the resulting solutions. However, this process does not guarantee that optimal solutions are achieved. For this purpose, the form-finding process could be automated by coupling the envelope grammar with an optimization algorithm, but at the expense of design flexibility and time. By giving the designer control over rule application, LIDIA permits the user to also meet other requirements, which could be disregarded in an automated process.

Using shape grammars as a generative system presents some advantages when compared to a parametric design system. First, it allows to define a more diversified universe of design solutions as it permits to encode topological variation, not just dimensional variation. Second it permits to obtain efficient designs in the capture of solar energy more quickly, as the shape transformation rules have embedded knowledge in this regard. The parametric design system could be coupled with an optimization algorithm, but this would be more time consuming and less flexible as mentioned above.

Future work will mainly concern two aspects, related with the short comings of the system. First, it will aim at improving the capabilities of the current system to relate the building envelope design with the design of interior spaces. Second, it will target the coupling of the generative design system with an optimization algorithm to facilitate the exploration and finding of solutions with improved energy production values. With these developments, the aim is to enhance the digital design of building envelopes and realize the potential of energy-based design, with the expectation that they can contribute to improving quality of life and reducing the ecological footprint of buildings, addressing climate change.

Acknowledgements. Daniel Mateus was supported with a grant (CIAUD_BI35/04008/2013) from CIAUD, Lisbon School of Architecture, University of Lisbon, Portugal. 


\section{References}

Beirão, J., Duarte, J.P. (2018). Generic grammars for design domains. Artificial Intelligence for Engineering Design, Analysis and Manufacturing, 32, 225-239.

Benrós, D. (2018). A generic housing grammar for the generation of different housing languages: A generic housing shape grammar for Palladian villas, Prairie and Malagueira houses. PhD dissertation. UCL Bartlett School of Graduate Studies, London, England.

Duarte, J.P. (2001). Customizing Mass Housing: A Discursive Grammar for Siza's Malagueira Houses. PhD dissertation. Massachusetts Institute of Technology, Cambridge, Massachusetts, United States of America.

Granadeiro, V. (2013). Early design and optimization of the building envelope: Integrating architectural generative design systems and energy performance calculation. PhD dissertation. Instituto Superior Técnico, Universidade Técnica de Lisboa, Lisboa, Portugal.

PORDATA. (2019). Consumo de energia elétrica per capita: total e por tipo de consumo. Retrieved June 12, 2019, from https://www.pordata.pt/DB/Portugal/Ambiente+de+Consulta/Tabela

Reis, P. (2018). Quais são os painéis solares fotovoltaicos mais eficientes no mercado? Retrieved June 12, 2019, from https://www.portal-energia.com/paineis-solaresfotovoltaicos-mais-eficientes-mercado/

Sagan, C. (1980). Cosmos. Random House.

Stiny, G. (1980). Introduction to shape and shape grammars. Environment and Planning $B, 7,343-351$.

Stiny, G. (1981). A note on the description of designs. Environment and Planning B, 8, 257-267.

United Nations Environment and International Energy Agency. (2017). Towards a zeroemission, efficient and resilient buildings and construction sector. Global Status Report 2017. 